\title{
Comparación de resultados de las tasas de filtración glomerular estimada por ecuaciones FAS y CKD-EPI en jóvenes estudiantes universitarios
}

\author{
Brissón, C.M. • Cuestas, V.I. • Denner, S.G. • Fernández, V.G • Prono Minella, P. del C. • Bonifacino \\ Belzarena, R. • Brissón, M.E. • Colussi, V.G. • Marsili, S.E. • Rosso, F.
}

Para citar este artículo: Brissón, C.M.; Cuestas, V.I.; Denner, S.G.; Fernández, V.G.; Prono Minella, P. del C.; Bonifacino Belzarena, R.; Brissón, M.E.; Colussi, V.G.; Marsili, S.E.; Rosso, F. (2021). Comparación de resultados de las tasas de filtrado glomerular estimada por ecuaciones FAS y CKD-EPI en jóvenes estudiantes universitarios. Pág. 13-26. Santa Fé, Argentina: UNL. DOI 10.14409/fabicib.v24i0.10748 


\section{COMPARACIÓN DE RESULTADOS DE LAS TASAS DE FILTRACIÓN GLOMERULAR ESTIMADA POR ECUACIONES FAS Y CKD-EPI EN JÓVENES ESTUDIANTES UNIVERSITARIOS}

Brissón, C.M.; Cuestas, V.I.; Denner, S.G.; Fernández, V.G; Prono Minella, P. del C.; Bonifacino Belzarena, R.; Brissón, M.E.; Colussi, V.G.; Marsili, S.E.; Rosso, F.

cmbrissons@gmail.com

Departamento de Bioquímica Clínica y Cuantitativa. Facultad de Bioquímica y Ciencias Biológicas. Universidad Nacional del Litoral. Ciudad Universitaria. S3000ZAA Santa Fe. Argentina. Tel.: (0342) 4575206 int. 185.

Recibido: 31/12/2020. Aceptado: 02/06/2021

\section{RESUMEN}

La tasa de filtración glomerular se estima por fórmulas (TFGe). CKD-EPI (válida de 18-70 años) es recomendada desde 2012 y FAS (Full age spectrum) propuesta en 2016, integra edades (2 años en adelante). Los valores de TFG se clasifican en categorías G. La categorización varía según fórmula empleada. La concordancia en asignación de la TFG a cada categoría es parte de evaluar el desempeño de las ecuaciones. Se analizaron, en jóvenes, diferencias medias entre TFGe por CKD-EPI y FAS y concordancia en asignación a categorías $\mathrm{G}$, total y según sexo. Estudio analítico. Muestra: 157 estudiantes voluntarios, 18-37 años. Creatininemia: método Jaffé cinético trazable a Isotopic Dilution Mass Spectroscopy. Estudio aprobado por Comité de Ética. Programa: MedCalc. CKD-EPI sobreestima a FAS en 7,6mL/min/1,73m² en el total y casi $50 \%$ más en mujeres que varones. FAS reclasifica $12,7 \%$ del total en G2 (TFG ligeramente disminuida) que CKD-EPI clasifica en G1 (TFG normal-alta), 8,0\% de varones, 13,7\% de mujeres. Se haIlaron indicios de ajuste diferente por sexo según ecuación, a confirmarse en una muestra con más varones. Identificar un buen método de estimación de TFG en adultos jóvenes es fundamental para detectar tempranamente enfermedad renal o evitar su sobrediagnóstico en este grupo etario.

Palabras claves: tasa de filtrado glomerular; FAS; CKD-EPI; comparación de métodos

El protocolo fue evaluado y aprobado por el Comité Asesor de Ética y Seguridad de la Investigación de la Facultad de Bioquímica y Ciencias Biológicas de la Universidad Nacional del Litoral, incluyó consentimiento informado y cuestionario sobre datos de historia clínica del estudiante y su familia, además de sus hábitos de vida. 


\section{SUMMARY}

\section{Comparison of results of glomerular filtration rates estimated by FAS and CKD-EPI equations in young university students}

The glomerular filtration rate (GFR) is estimated using formulas. CKD-EPI (valid from 18 to 70 years old) has been recommended since 2012 and FAS (Full age spectrum) was proposed in 2016 to integrate ages from 2 years onwards. The GFR values are classified in categories G. Categorization varies according to the formula. The agreement of GFR assignment to each category is part of the evaluation of the equations performance. The differences between GFRs estimated by CKD-EPI and FAS and their concordance in the assignment to $\mathrm{G}$ categories were evaluated in young students, total and according to sex. Analytical study. Sample: 157 volunteer students, 18-37 years old. Creatininemia: kinetic Jaffé method traceable to Isotopic Dilution Mass Spectroscopy. The study was approved by the Ethics Committee. Program: MedCalc. CKD-EPI overestimates FAS by $7.6 \mathrm{~mL} / \mathrm{min} / 1.73 \mathrm{~m}^{2}$ in the total and almost $50 \%$ more in women than in men. FAS reclassifies $12.7 \%$ of the total in G2 (slightly decreased GFR) than CKD-EPI classifies in G1 (norm-high GFR), $8.0 \%$ of men, $13.7 \%$ of women. Clues for different adjustment by sex were found according to the equation that have to be confirmed in a larger sample, with more men. A good TFG estimation method identification in young adults is fundamental to detect kidney disease early in this age group and to avoid its overdiagnosis.

\section{INTRODUCCIÓN}

En 2002 se define la Enfermedad Renal Crónica (ERC), considerada un problema mundial de salud pública en base a la prevalencia surgida de los estudios epidemiológicos. Desde entonces se ha avanzado mucho en el campo de diseño de fórmulas de estimación de la tasa de filtración glomerular (TFG). La TFG mide la función renal de filtración en salud y enfermedad renal. Es el indicador usado, solo o junto a otros marcadores de daño renal, en la definición de la ERC. La ERC se define en pacientes con TFG $\geq 60 \mathrm{~mL} / \mathrm{min} / 1,73 \mathrm{~m}^{2}$ con marcadores de daño renal persistentes durante más de 3 meses y en pacientes con TFG $<60 \mathrm{~mL} / \mathrm{min} / 1,73 \mathrm{~m}^{2}$, también por más de 3 meses y, en ambos casos con compromiso para la salud. (1)

La medición de la TFG, hecha por el método de referencia, depuración de inulina, u otros de similar categoría validados frente al mismo, tales como ${ }^{125}$-lothalamato, lohexol, ${ }^{51} \mathrm{Cr}$-EDTA, DTPA (ácido dietilentriaminopentaacético) generalmente marcado con ${ }^{99 \mathrm{~m}} \mathrm{Tc}$ son poco prácticos en la rutina de nefrología y menos todavía para usarse en estudios epidemiológicos. Se han realizado muchos esfuerzos para encontrar maneras de estimarla sin recurrir a ellos. De uso corriente es el clearance de creatinina, con la dificultad asociada a la recolección de la orina de $24 \mathrm{~h}$ y a que la creatinina no es solamente filtrada por el riñón sino que también se 
secreta, por lo que no tiene las mismas particularidades como molécula que la inulina para evaluar la TFG. Se han desarrollado múltiples fórmulas para estimar la TFG (TFGe). La mayoría de las guías clínicas recomienda su uso. Kidney Disease: Improving Global Outcomes (KDIGO) en 2012 (KDIGO 2012), sugiere usar la ecuación Chronic Kidney Disease Epidemiology (CKD-EPI) en individuos de 18 a 70 años que se encuentren dentro de las condiciones de aplicar la fórmula. (1)(2)

Existen fórmulas para niños y adolescentes, para adultos entre 18 y 70 años, para mayores de 70 años. Las variaciones que surgen según el modo de estimación al cambiar de grupo etario pueden generar dificultades si se está siguiendo a un paciente con patología renal. Para solucionar estas discontinuidades, Pottel en 2016, modeló una ecuación a la que denominó FAS, Full Age Spectrum, de uso a partir de los dos años de edad. (3)

\section{CKD-EPI}

$T F G=141 \times \min (C r / K, 1)^{\alpha} \times \max (C r / K, 1)^{-1,209} \times 0,99^{\text {Edad }} \times 1,018$ [si es mujer] $\times 1,159$ [si es negro] $\mathrm{Cr}$ : creatinina sérica en $\mathrm{mg} / \mathrm{dL}, \mathrm{k}: 0,7$ (mujeres) y 0,9 (varones); $\alpha$ : -0,329 (mujeres) y -0,411 (varones); $\mathrm{min}$ indica el mínimo entre $\mathrm{Cr} / \kappa$ o 1, y max indica el máximo entre $\mathrm{Cr} / \mathrm{k}$ o 1.

\section{FAS}

$$
\begin{array}{ll}
2 \text { años } \leq \text { edad } \leq 40 \text { años } & T F G e=\frac{107,3}{C r / Q c r e} \\
>40 \text { años } & \text { TFGe }=\frac{107,3}{C r / Q c r e} \times 0,988^{(\text {edad }-40)}
\end{array}
$$

Qcre: mediana de la Cr para una determinada edad y sexo en población sana

La TFG medida o estimada, se clasifica según KDIGO 2012, en categorías G que se usan para describir su nivel independientemente de la presencia o ausencia de enfermedad renal. (1)(4)

\begin{tabular}{|c|c|c|}
\hline Categoría & Función renal & TFG $\left(\mathrm{mL} / \mathrm{min} / 1,73 \mathrm{~m}^{2}\right)$ \\
\hline G1 & Normal o alta & $\geq 90$ \\
\hline $\mathbf{G 2}$ & Levemente disminuida & $60-89$ \\
\hline G3a & Disminución leve a moderada & $45-59$ \\
\hline G3b & Disminución moderada a severa & $30-44$ \\
\hline G4 & Disminución severa & $15-29$ \\
\hline G5 & Fallo renal & $<15$ \\
\hline
\end{tabular}

Tabla 1. Categorías de G de TFG

Fuente: traducido por los autores de Kidney Disease: Improving Global Outcomes (KDIGO) CKD Work Group., 2013. KDIGO 2012 Clinical Practice Guideline for the Evaluation and Management of Chronic Kidney Disease. Kidney IntSuppl. 3,1:1-150

La fórmula óptima para estimar la TFG es la que obtenga el mismo valor que la TFG medida en todo el rango de referencia. Esta ecuación no ha sido hallada todavía y la mayoría ajusta mejor para valores $<60$ $\mathrm{mL} / \mathrm{min} / 1,72 \mathrm{~m}^{2}$. Una característica importante clínica y epidemiológicamente, es que clasifiquen al mismo individuo en la misma categoría G. Las categorías G1 y G2, que representan valores de TFG normales a altos y 
levemente disminuidos, respectivamente, no pueden diferenciarse entre sí con muchas de las fórmulas habitualmente usadas debido a las restricciones asociadas a su modelado. De esta forma, se pueden asignar a esas categorías solamente las TFG medidas con métodos de referencia, las determinadas por clearance de creatinina y las estimadas con algunas ecuaciones como CKD-EPI y FAS.

Las ecuaciones deben validarse si se van a aplicar en poblaciones diferentes a aquéllas en que fueron diseñadas. Se consideró importante evaluar su comportamiento relativo en jóvenes en las categorías G1 y G2 de TFG para agregar evidencia sobre su desempeño en este grupo. Esto aportaría al conocimiento de cómo estima FAS la TFG respecto a la recomendada entre los 18 y los 70 años, CKD-EPI, en individuos que reúnen características donde las ecuaciones presentan dificultades sobre las que la comunidad científica está trabajando en superar: edad en rango próximo a los límites de su aplicación y con TFG superiores a 60 $\mathrm{mL} / \mathrm{min} / 1,73 \mathrm{~m}^{2}$

Cabe aclarar que en este trabajo se usó la fórmula de Pottel anterior a su modificación publicada en 2020 y que dio origen a una nueva ecuación. (5)

\section{OBJETIVO}

Evaluar las diferencias medias entre la TFG estimada por CKD-EPI y FAS y la concordancia de ambas ecuaciones en la asignación a las categorías G de TFG en una muestra de jóvenes estudiantes, para el total y según sexo.

\section{MATERIAL Y MÉTODO}

Estudio analítico de corte transversal. Participaron 157 estudiantes voluntarios de las carreras de Bioquímica y de Licenciatura en Nutrición de la Universidad Nacional del Litoral (UNL) de la República Argentina, en el período comprendido entre noviembre de 2014 y noviembre de 2019. Todos ambulatorios. Por restricciones en el uso de CKD-EPI no se incluyeron embarazadas, amputados ni afectados por enfermedades consuntivas, patologías agudas, en tratamiento con diuréticos, vegetarianos o voluntarios con índice de masa corporal (IMC) extremos $\left(<18 \mathrm{Kg} / \mathrm{m}^{2} \mathrm{o} \geq 35 \mathrm{Kg} / \mathrm{m}^{2}\right)$. Se utilizó un Cuestionario de Datos Generales e Historia Clínica con el que se obtuvo información de antecedentes personales y familiares relacionados a patologías renales o que pudieran tener impacto renal, hábitos de vida y medicación. A cada voluntario se le tomaron medidas antropométricas: peso y talla para calcular el IMC. Se les extrajo sangre en ayunas para determinar la creatininemia. La creatinina se midió por método Jaffé cinético trazable a Isotopic Dilution Mass Spectroscopy (IDMS) en autoanalizador Cobas c111 (Roche Diagnostics Ltd. Rotkreuz, Suiza).

Se calcularon las TFGe por CKD-EPI y FAS y se clasificaron en categorías G. 
El protocolo fue evaluado y aprobado por el Comité Asesor de Ética y Seguridad de la Investigación de la Facultad de Bioquímica y Ciencias Biológicas de la UNL, incluyó consentimiento informado y cuestionario sobre datos de historia clínica del estudiante y su familia, además de sus hábitos de vida.

Estadística: Para el análisis estadístico descriptivo se calcularon media, mediana, desviación estándar y rango intercuartílico, de las variables edad y TFGe por CDK-EPI y FAS, para todo el rango de TFG y por categoría G, para el grupo total de casos y según el sexo. Se evaluó mediante el test de Shapiro-Wilk si las distribuciones de los datos eran o no normales, aplicando luego pruebas paramétricas o no, según correspondiere. Para el análisis de las diferencias entre datos pareados se usó el t-test para muestras pareadas. Se empleó la prueba de MannWhitney para evaluar si existían diferencias entre las edades según sexo. La concordancia de asignación a categoría G por ambas ecuaciones se evaluó mediante el índice Kappa con valoración de Landis y Koch (6).

Tabla 2. Valoración del coeficiente Kappa

\begin{tabular}{|c|c|}
\hline Coeficiente Kappa & Fuerza de concordancia \\
\hline 0,00 & Pobre \\
\hline $0,01-0,20$ & Leve \\
\hline $0,21-0,40$ & Aceptable \\
\hline $0,41-0,60$ & Moderada \\
\hline $0,61-0,80$ & Considerable \\
\hline $0,81-1,00$ & Casi perfecta \\
\hline
\end{tabular}

Fuente: Landis J, Koch G: The measurement of observer agreement for categorical data. Biometrics. 1977; 33: 159-174.

Se compararon los resultados de TFGe por CDK-EPI y FAS mediante el análisis de Bland-Altman; los gráficos se generaron relacionando la diferencia CKD-EPI - FAS con la media de las dos estimaciones. En todos los casos se utilizó un nivel de confianza del 95\%, p<0,05. Programas estadísticos: Microsoft Excel, MedCalc statistical software versión libre.

\section{RESULTADOS}

De los 157 participantes, 25 fueron varones y 132 mujeres. La composición por sexo de la muestra refleja la de las carreras a las que pertenecen los estudiantes voluntarios.

Las edades de todo el grupo estuvieron comprendidas entre 18 y 37 años (24,95 $\pm 3,00$ años). Para los varones el rango etario fue de 19 a $37(25,96 \pm 3,42$ años) y para las mujeres 18 a $36(24,76 \pm 2,88$ años). Se comprobó que el promedio de las edades de varones y mujeres no difería significativamente (prueba de Mann-Whitney; $p=0,0587)$.

En el Gráfico 1 se observa la distribución de las TFGe por cada fórmula. 
Gráfico 1. Distribución de las TFGe según CKD-EPI y FAS ( $n=157)$.

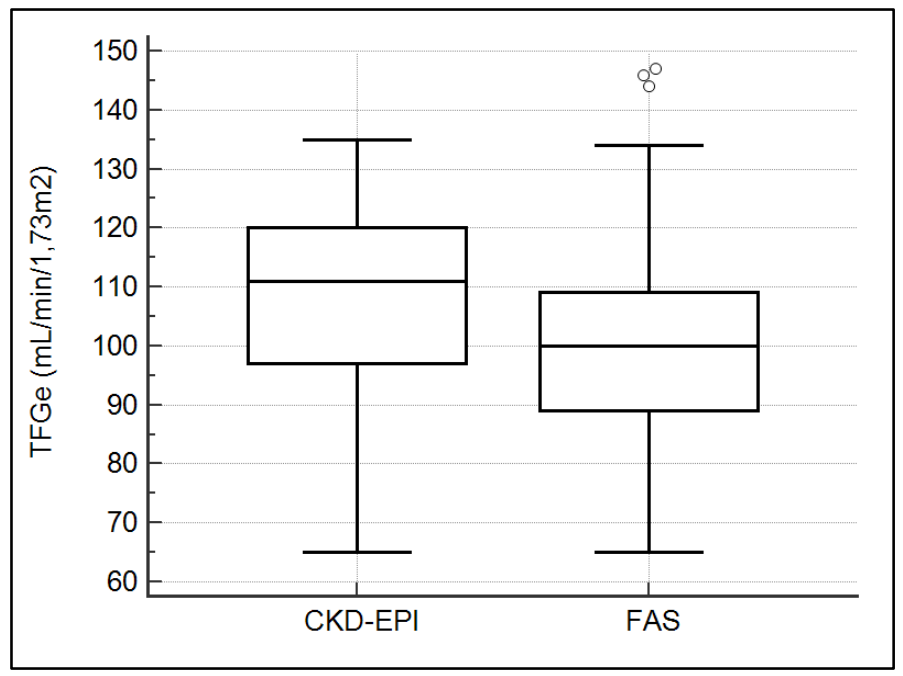

Fuente: elaboración a partir de resultados del proyecto

El análisis mostró la mediana de TFGe por FAS menor que la de CKD-EPI y una distribución de las TFGe por FAS más simétrica que la de CKD-EPI.

En la Tabla 3 se pueden ver los resultados de la TFGe por cada ecuación abarcando todos los niveles de la misma y por categoría G, para el total y por sexo.

Tabla 3. Tasa de filtrado glomerular según método de estimación, total, por sexo y por categoría G.

\begin{tabular}{|c|c|c|c|c|}
\hline \multirow{2}{*}{$\begin{array}{c}\text { Categoría } \\
\text { G }\end{array}$} & \multirow{2}{*}{$\begin{array}{l}\text { Estimador } \\
\text { de TFG }\end{array}$} & Total & Varones & Mujeres \\
\hline & & $\begin{array}{c}\text { Media } \pm \text { DS* } \\
\text { (n) }\end{array}$ & $\begin{array}{c}\text { Media } \pm D S^{*} \\
\text { (n) }\end{array}$ & $\begin{array}{c}\text { Media } \pm \text { DS * } \\
\text { (n) }\end{array}$ \\
\hline \multirow[t]{2}{*}{ Todas } & CKD-EPI & $\begin{array}{c}107,71 \pm 15,02 \\
(157)\end{array}$ & $\begin{array}{c}108,44 \pm 17,07 \\
(25)\end{array}$ & $\begin{array}{c}107,58 \pm 14,67 \\
(132)\end{array}$ \\
\hline & FAS & $\begin{array}{c}100,10 \pm 14,88 \\
(157)\end{array}$ & $\begin{array}{c}104,16 \pm 18,95 \\
(25)\end{array}$ & $\begin{array}{c}99,32 \pm 13,94 \\
(132)\end{array}$ \\
\hline \multirow[t]{2}{*}{ G1 } & CKD-EPI & $\begin{array}{c}111,62 \pm 11,34 \\
(137)\end{array}$ & $\begin{array}{c}113,80 \pm 11,97 \\
(21) \\
\end{array}$ & $\begin{array}{c}111,23 \pm 11,23 \\
(116)\end{array}$ \\
\hline & FAS & $\begin{array}{c}106,02 \pm 11,60 \\
(117)\end{array}$ & $\begin{array}{c}111,42 \pm 15,18 \\
(19)\end{array}$ & $\begin{array}{c}105,28 \pm 10,45 \\
(98)\end{array}$ \\
\hline \multirow[t]{2}{*}{ G2 } & CKD-EPI & $\begin{array}{c}80,95 \pm 8,22 \\
(20)\end{array}$ & $\begin{array}{c}80,25 \pm 10,68 \\
(4)\end{array}$ & $\begin{array}{c}81,12 \pm 7,91 \\
(16)\end{array}$ \\
\hline & FAS & $\begin{array}{c}81,87 \pm 6,61 \\
(40)\end{array}$ & $\begin{array}{c}81,17 \pm 7,70 \\
(6)\end{array}$ & $\begin{array}{c}82,18 \pm 6,48 \\
(34)\end{array}$ \\
\hline
\end{tabular}

Nota. ${ }^{*} \mathrm{~mL} / \mathrm{min} / 1,73 \mathrm{~m}^{2}$. Fuente: elaboración a partir de resultados del proyecto.

Para comparar las estimaciones entre fórmulas para todo el rango de TFG se usaron pruebas para muestras pareadas. La media de las diferencias de las TFGe por CKD-EPI y FAS fue distinta de cero para el nivel de significación escogido para todo el grupo y por sexo (t-student para datos pareados; $p<0,0001$ para el total del grupo y para las mujeres; $p=0,0087$ para varones). 
En la tabla 4 se presentan los resultados de las medias de las diferencias entre las TFGe por ambas ecuaciones (CKD-EPI - FAS) para el total de estudiantes y por sexo.

Tabla 4. Medias de las diferencias (CKD-EPI - FAS) e IC $95 \%$ entre los pares de valores de TFG obtenidos mediante las ecuaciones CKDEPI y FAS, general y por sexo.

\begin{tabular}{|c|c|c|}
\hline \multicolumn{2}{|c|}{ Media de las diferencias entre estimaciones (CKD-EPI - FAS) (IC95\%) } \\
$\mathbf{m L} / \mathbf{m i n} / \mathbf{1}, \mathbf{7 3} \mathbf{~ m}^{\mathbf{2}}$
\end{tabular}

Nota: diferencias medias e $\mathrm{IC}_{95 \%}$ en $\mathrm{mL} / \mathrm{min} / 1,73 \mathrm{~m}^{2}$. Fuente: elaboración a partir de resultados del proyecto

Se determinó que CKD-EPI sobreestima a FAS en unos $7,6 \mathrm{~mL} / \mathrm{min} / 1,73 \mathrm{~m}^{2}$ en general y que en varones esta sobreestimación es casi la mitad que en las mujeres, aproximándose para ese grupo los valores obtenidos con ambas fórmulas.

En los Gráficos 2, 3 y 4 la concordancia evaluada por el análisis de Bland-Altman muestra la distribución de la diferencia de los valores de TGFe por cada fórmula en función de su promedio para todos los estudiantes, para los varones y para las mujeres.

Gráfico 2. Gráfico de Bland-Altman para CKD-EPI y FAS en el total de la muestra $(n=157)$

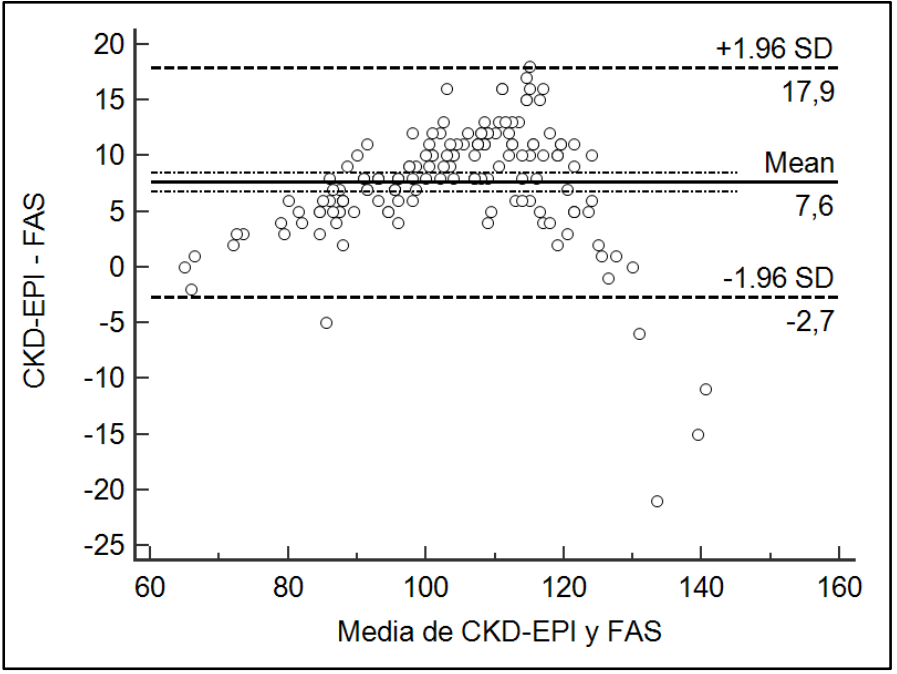

Fuente: elaboración a partir de resultados del proyecto 
Gráfico 3. Gráfico de Bland-Altman para CKD-EPI y FAS en varones ( $n=25)$

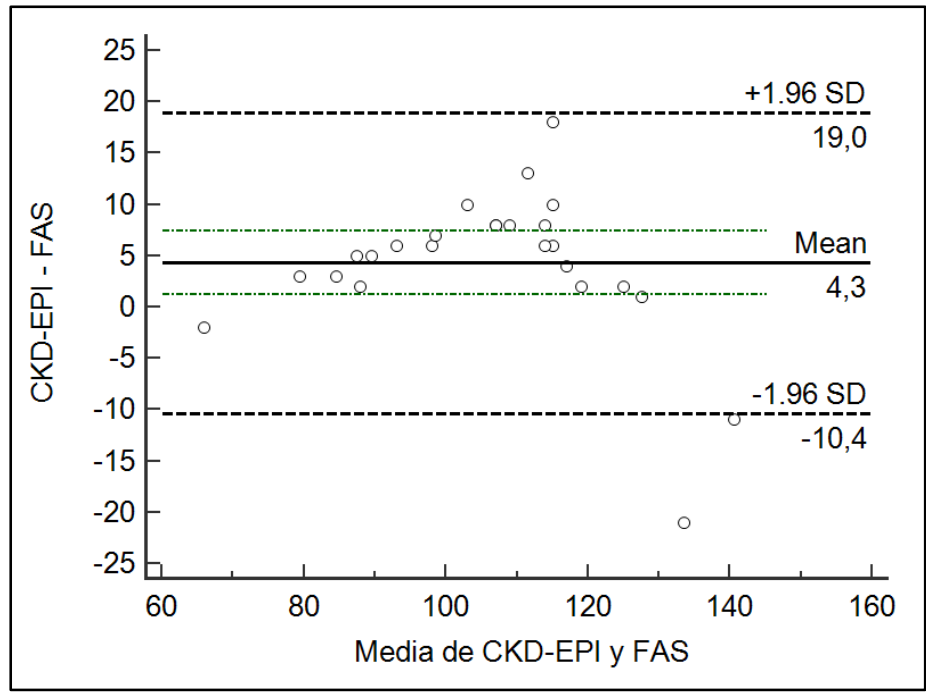

Fuente: elaboración a partir de resultados del proyecto

Gráfico 4. Gráfico de Bland-Altman para CKD-EPI y FAS en mujeres ( $n=132)$

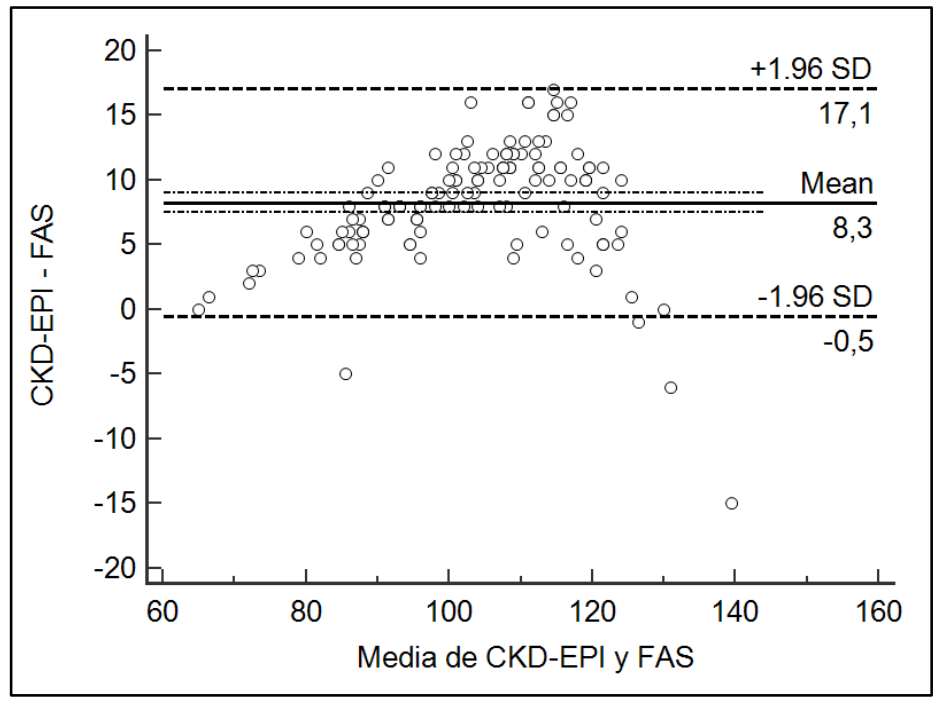

Fuente: elaboración a partir de resultados del proyecto

El análisis expresó gráficamente la sobreestimación de la TFGe por CKD-EPI respecto a FAS, que fue más acentuada en mujeres que en varones y que para valores altos de TFG la diferencia cambió de signo, superando las estimaciones por FAS a las estimaciones por CKD-EPI.

En la tabla 5 se muestra la concordancia en asignación a las categorías G1 (TFGe $\geq 90 \mathrm{~mL} / \mathrm{min} / 1,73 \mathrm{~m}^{2}$, normal a alta) y G2 (TFGe $60-89 \mathrm{~mL} / \mathrm{min} / 1,73 \mathrm{~m}^{2}$, ligeramente disminuida) entre los dos métodos usados para estimar la TFG de acuerdo a los valores del índice Kappa. 
Tabla 5. Índice Kappa de concordancia en la asignación a categoría G entre CKD-EPI y FAS, IC95\% y fuerza de concordancia según Landis y Koch, general y por sexo.

\begin{tabular}{|l|l|l|}
\hline Grupo & Kappa(IC $\left.\mathbf{C}_{9 \%}\right)$ & Fuerza de concordancia \\
\hline General & $0,598(0,448-0,749)$ & Moderada a considerable \\
\hline Varones & $0,752(0,434-1,000)$ & Moderada a casi perfecta \\
\hline Mujeres & $0,569(0,402-0,736)$ & Aceptable a considerable \\
\hline
\end{tabular}

Fuente: elaboración a partir de resultados del proyecto

Se observó que la fuerza de concordancia entre las ecuaciones de estimación para asignar a categoría G era mayor para los varones que para las mujeres pero con un intervalo de confianza más amplio.

En el gráfico 5 se pueden observar las proporciones de varones y mujeres clasificadas en G1 y G2 por cada ecuación.

Gráfico 5. Proporción de varones y mujeres con TFGe clasificada en G1 y G2 según CKD-EPI y según FAS.

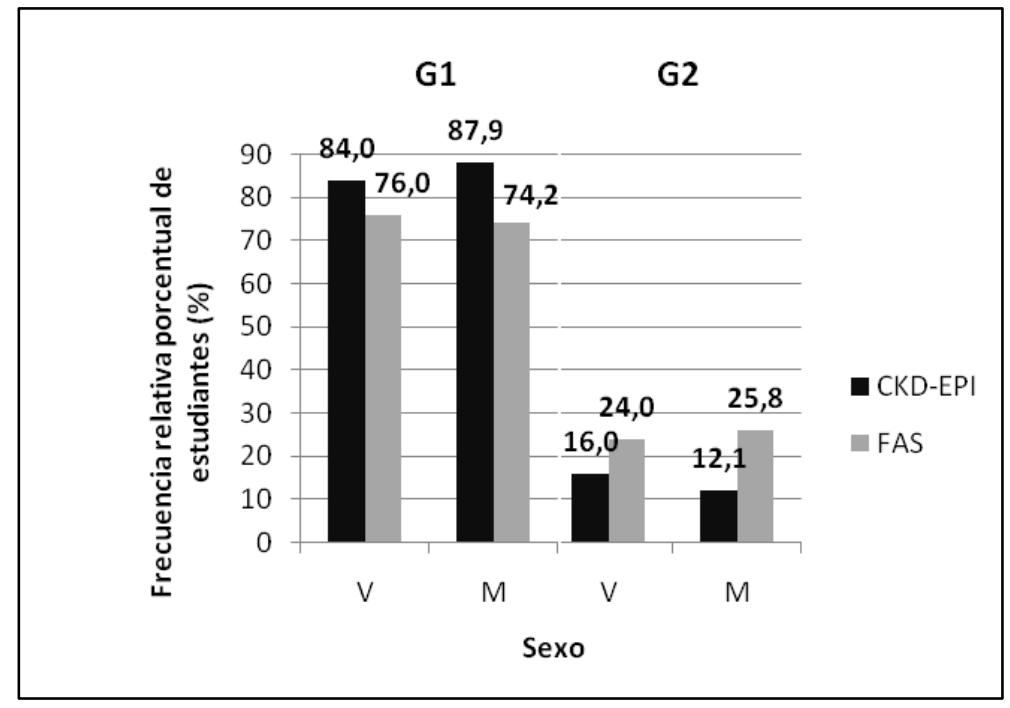

Nota. V: varones; M: mujeres; G1: TFGe $\geq 90 \mathrm{~mL} / \mathrm{min} / 1,73 \mathrm{~m}^{2} ; \mathrm{G} 2: \mathrm{TFGe}=60-89 \mathrm{~mL} / \mathrm{min} / 1,73 \mathrm{~m}^{2}$. Fuente: elaboración a partir de resultados del proyecto

Al estimar con CKD-EPI, el 84\% de los varones y casi el 88\% de las mujeres tenían TFGe en la categoría G1. Al estimar con FAS los porcentajes bajaron, pasando el $8 \%$ de los varones y el $13,7 \%$ de las mujeres a la categoría G2. En el gráfico 5 se pudieron observar estas diferencias de reclasificación donde al utilizar FAS, la proporción de mujeres que se reclasifican desde la categoría de TFG normal a alta (G1) a ligeramente disminuida 
(G2) es mayor que la de varones. Si bien la diferencia no fue significativa al nivel de confianza adoptado (prueba chi-cuadrado; $p>0,05)$, sabiendo que existe, habría que considerarla como variable de control en análisis multivariados posteriores.

\section{DISCUSIÓN}

Se sabe que, en jóvenes adultos que es el grupo de interés de esta investigación, CKD-EPI sobreestima a la TFG medida por métodos de referencia. Para el rango etario de 20 a 39 años, Selistre reporta que CKD-EPI sobreestima a la TFG medida un 14\% en general, un 32\% en G2 y un 7\% en G1. (7) Pottel halló que CKD-EPI sobreestima la TFG medida en unos $21 \mathrm{~mL} / \mathrm{min} / 1,73 \mathrm{~m}^{2}$ entre los 18 y 20 años, $19 \mathrm{~mL} / \mathrm{min} / 1,73 \mathrm{~m}^{2}$ entre los 20 y 24 años y 9,5 mL/min/1,73 $\mathrm{m}^{2}$ entre los 24 y 30 años y sugiere que no debería utilizase en adultos jóvenes. Para FAS la sobreestimación de la TFG medida es de 8,1 y $7,8 \mathrm{~mL} / \mathrm{min} / 1,73 \mathrm{~m}^{2}$ de 18 a 24 y de 24 a 30 años respectivamente. (8)

Björk, comparando CKD-EPI y FAS reporta para el rango de 20 a 39 años, un bias entre ambas de 4,7 $\mathrm{mL} / \mathrm{min} / 1,73 \mathrm{~m}^{2}$ en $\mathrm{G} 2$ y de $6 \mathrm{~mL} / \mathrm{min} / 1,73 \mathrm{~m}^{2}$ cuando la TFG medida estaba entre 90 y $119 \mathrm{~mL} / \mathrm{min} / 1,73 \mathrm{~m}^{2}$.(9) Estas diferencias son menores a las observadas en nuestra muestra pero en el mismo sentido. Debe tenerse en cuenta que, para aplicar FAS en la muestra se usaron los valores del coeficiente Qcre dados por Pottel debido a que no se disponen de valores locales para este parámetro.

En el caso de los estudiantes de Santa Fe no se hallaron TFGe que clasificaran fuera de G1 y G2 por ninguna de las dos fórmulas. Al utilizar FAS la cantidad de estudiantes en G2 es el doble que empleando CKD-EPI, 25,4\% vs. $12,7 \%$. El cambio de categoría al reclasificar según fórmula es más importante proporcionalmente para las mujeres que para los varones, aunque no resultara significativa la diferencia, se observó esta tendencia en todos los aspectos analizados.

En el estudio SCOPE (Screening for Chronic Kidney Disease among Older People across Europe) se compararon las mismas ecuaciones en individuos con una edad media de 80,3 años con TFG en categorías G 1 a 4. La diferencia hallada entre las TFGe por las mismas fórmulas fue, para el total de los individuos de 8,72 $\mathrm{mL} / \mathrm{min} / 1,73 \mathrm{~m}^{2}$. Los individuos clasificados en G1 por CKD-EPI representaron el 1,9\% y el 1,3\% por FAS. Al diferenciar según sexo, el 2,5\% de las mujeres y el 1,1\% de los hombres se hallaba en G1 por CKD-EPI mientras que por FAS lo hacía el 1,4\% de las mujeres no variando el porcentaje de hombres.

Esta diferencia en la clasificación se observó en las otras categorías: 43\% de los pacientes clasificados en G2 por CKD-EPI resultaron clasificados en G3a por FAS y 50\% de los individuos clasificados en categoría G3a por CKD-EPI fueron clasificados en G3b por FAS. El coeficiente Kappa obtenido fue de 0,47 para el grupo completo, 0,36 para mujeres y 0,57 para varones y concluyeron que el desacuerdo entre CKD-EPI y FAS era más evidente entre las mujeres que entre los hombres. (10) Esta tendencia, en el bias entre las ecuaciones, en los cambios de proporciones en las categorías y en la evaluación de los coeficientes Kappa, se observa también 
en nuestra muestra por lo que podría no deberse a influencia de la diferencia de edad entre las muestras, aunque debe tenerse en cuenta la diferencia de tamaño de las muestras comparadas.

Sintetizando, CKD-EPI que según KDIGO 2012 debe usarse para calcular la TFGe entre los 18 y 70 años, sobreestima los valores hallados en los adultos jóvenes respecto a la medida con métodos de referencia. Se están desarrollando nuevas ecuaciones para disminuir este sesgo que impacta en la categoría G de TFG, clasificando a muchos individuos de este grupo etario en categorías de mejor función renal de filtración que las que tendrían si se midiera. Como otros autores, hallamos que CKD-EPI sobreestima a FAS excepto a valores altos de TFG. Esta diferencia repercute en la concordancia entre ambos estimadores en la asignación a las categorías $\mathrm{G}$ y una proporción de jóvenes se clasifican en categoría de menor TFG con FAS que con CKD-EPI.

Limitaciones: la comparación entre las ecuaciones no incluye una comparación frente a un método de referencia de medida de TFG. Los hallazgos por sexo deben ser confirmados en una muestra con mayor número de varones. La muestra no es aleatoria pero se considera que la información aportada para describir las categorías G1 y G2 en jóvenes y mostrar la diferencia de comportamiento de los estimadores de TFG usadas en este grupo etario es valiosa debido a que no existen motivos esenciales que diferencien a los voluntarios de los individuos que conforman la población de estudiantes.

\section{CONCLUSIONES}

En la muestra, los valores de TFGe por FAS fueron menores que por CKD-EPI que es la recomendada por KDIGO 2012 para el rango etario estudiado. Esto afecta la clasificación por categoría G. Así, la TFGe de un 12,7\% de los jóvenes resultó ligeramente disminuida al estimarse con FAS en lugar de normal a alta como resultaba con CKDEPI. Se ven mayores diferencias en las mujeres que en los varones con mayor reclasificación proporcional de las primeras de G1 a G2 al estimar por FAS y menor fuerza de concordancia que en varones. Si bien los varones en la muestra son pocos, se perfila un ajuste diferente por sexo entre ambas ecuaciones, tendencia que debe confirmarse en mayor número de individuos. Estos resultados representan una base para la evaluación, en la misma muestra, del ajuste de la FAS modificada en 2020. Es de importancia toda información sobre las características de los métodos de estimación de la TFG en valores superiores a $60 \mathrm{~mL} / \mathrm{min} / 1,73 \mathrm{~m}^{2}$ y en adultos jóvenes, a efectos de solucionar el más adecuado para uso clínico y tamizaje poblacional, tanto para detectar tempranamente ERC en este grupo etario como para evitar su sobrediagnóstico.

Proyectos financiados por el Programa CAI+D de la Universidad Nacional del Litoral. República Argentina: “Enfermedad renal crónica en estudiantes de Bioquímica de la UNL: detección, prevalencia y biomarcadores emergentes de daño renal. 2013-2016". CAl+D 2011. 50120110100130 Ll y “Estadios tempranos asintomáticos de enfermedad renal crónica en estudiantes de la UNL: prevalencia, comportamiento de estimadores de la función renal, caracterización de marcadores de daño renal y del riesgo cardiovascular". 2017-2019." CAI+D 2016. 50120150100022LI. 


\section{BIBLIOGRAFÍA}

1-Kidney Disease: Improving Global Outcomes (KDIGO) CKD Work Group.,2013. KDIGO 2012 Clinical Practice Guideline for the Evaluation and Management of Chronic Kidney Disease. Kidney Int Suppl. 3,1:1-150. Disponible en https://www.sciencedirect.com/journal/kidney-international-supplements/vol/3/issue/1 2-Levey, A.S.; Stevens, L.A.; Schmid, C.H.; Zhang, Y.; Castro, A.F.; Fieldman, H.I.; Kusek, J.W.; Eggers, P.; Van Lente, F.; Greene, T.; Coresh, J., 2009. A new equation to estimate glomerular filtration rate. Ann Intern Med. 150, 9:604-612. Disponible en https://pubmed.ncbi.nlm.nih.gov/19414839/

3-Pottel, H.; Hoste, L.; Dubourg, L.; Ebert, N.; Schaeffner, E.; Eriksen, B.O.; Melsom, T.; Lamb, E.J.; Rule, A.D.; Turner. S.T.; Glassock, R.J.; De Souza, V.; Selistre, L.; Mariat, C.; Martens, F.; Delanaye, P., 2016. An estimated glomerular filtration rate equation for the full age spectrum. Nephrol Dial Transplant. 31, 5: 798-806. Disponible en https://pubmed.ncbi.nlm.nih.gov/26932693/

4-Levey, A.S.; Eckardt, K.U.; Dorman, N.M.; Christiansen, S.L.; Hoorn, E.J.; Ingelfinger, J.R.; Inker, L.A.; Levin, A.; Mehrotra, R.; Palevsky, P.M.; Perazella, M.A.; Tong, A.; Allison, S.J.; Bockenhauer, D.; Briggs, J.P.; Bromberg, J.S.; Davenport, A.; Feldman, H.I.; Fouque, D.; Gansevoort, R.T.; Gill, J.S.; Greene, E.L.; Hemmelgarn, B.R.; Kretzler, M.; Lambie, M.; Lane, P.H.; Laycock, J.; Leventhal, S.E.; Mittelman, M.; Morrissey, P.; Ostermann, M.; Rees, L.; Ronco, P.; Schaefer, F.; St Clair Russell, J.; Vinck, C.; Walsh, S.B.; Weiner, D.E.; Cheung, M.; Jadoul, M.; Winkelmayer,W.C., 2020. Nomenclature for kidney function and disease: report of a kidney disease: improving global outcomes (KDIGO) consensus conference. Kidney Int. 97, 6:1117-1129. Disponible en https://doi.org/10.1177/0896860820934730

5-Pottel, H.; Björk, J.; Courbebaisse, M.; Couzi, L.; Ebert, N.; Eriksen, B.O.; Dalton, R.N.; Dubourg, L.; Gaillard, F.; Garrouste, C.; Grubb, A.; Jacquemont, L.; Hansson, M.; Kamar, N.; Lamb, E.J.; Legendre, C.; Littmann, K.; Mariat, C.; Melsom, T.; Rostaing, L.; Rule, A.D.; Schaeffner, E.; Sundin, P.O.; Turner, S.; Bökenkamp, A.; Berg, U.; Åsling-Monemi, K.; Selistre, L.; Åkesson, A.; Larsson, A.; Nyman, U.; Delanaye, P., 2020. Development and validation of a modified full age spectrum creatinine-based equation to estimate glomerular filtration rate. A cross-sectional analysis of pooled data. Ann Intern Med. Epub ahead of print. Disponible en https://www.acpjournals.org/doi/10.7326/M20-4366

6-Landis, J.; Koch, G., 1977. The measurement of observer agreement for categorical data. Biometrics. 33, 1: 159-74.

7-Selistre, L.; Rabilloud, M.; Cochat, P.; de Souza, V.; Iwaz, J.; Lemoine, S.; Beyerle, F.; Poli-de-Figueiredo, C.E.; Dubourg, L., 2016. Comparison of the Schwartz and CKD-EPI Equations for Estimating Glomerular Filtration Rate in Children, Adolescents, and Adults: A Retrospective Cross-Sectional Study. PLoSMed. 13, 3:e1001979. Disponible en https://www.ncbi.nlm.nih.gov/pmc/articles/PMC4811544/

8-Pottel, H.; Björk, J.; Bökenkamp, A.; Berg, U.; Åsling-Monemi, K.; Selistre, L.; Dubourg, L.; Hansson, M.; Littmann, K.; Jones, I.; Sjöström, P.; Nyman, U.; Delanaye, P., 2019. Estimating glomerular filtration rate 
at the transition from pediatric to adult care. Kidney Int. 95, 5:1234-1243. Disponible en https://pubmed.ncbi.nlm.nih.gov/30922665/

9-Björk, J.; Nyman, U.; Courbebaisse, M.; Couzi, L.; Dalton, R.N.; Dubourg, L.; Ebert, N.; Eriksen, B.O.; Gaillard, F.; Garrouste, C.; Grubb, A.; Hansson, M.; Jacquemont, L.; Jones, I.; Kamar, N.; Lamb, EJ.; Legendre, C.; Littmann, K.; Mariat, C.; Melsom, T.; Rostaing, L.; Rule, A.D.; Schaeffner, E.; Sundin, P.; Turner, S.; Åkesson, A.; Delanaye, P.; Pottel, H., 2020. Prospects for improved glomerular filtration rate estimation based on creatinine-results from a transnational multicentre study. Clin Kidney J. 13, 4: 674-683. Disponible en https://academic.oup.com/ckj/article/13/4/674/5857035

10-Corsonello, A.; Roller-Wirnsberger, R.; Wirnsberger, G.; Ärnlöv, J.; Carlsson, A.C.; Tap, L.; Mattace-Raso, F.; Formiga, F.; Moreno-Gonzalez, R.; Weingart, C.; Sieber, C.; Kostka, T.; Guligowska, A.; Gil, P.; Lainez Martinez, S.; Artzi-Medvedik, R.; Melzer, I.; Lattanzio, F., 2020. Clinical Implications of Estimating Glomerular Filtration Rate with Three Different Equations among Older People. Preliminary Results of the Project "Screening for Chronic Kidney Disease among Older People across Europe (SCOPE)". J. Clin. Med. 9, 294. Disponible en https://pubmed.ncbi.nlm.nih.gov/31973029/

\section{Agradecimiento}

A los estudiantes que voluntariamente participaron.

Los resultados fueron presentados en forma preliminar y parcial en el Congreso Nacional Bioquímico CUBRA 2019. Resistencia, Chaco, 25, 26 y 27 de septiembre del 2019 